\title{
comment
}

\section{Towards a more inclusive outreach}

\author{
Scientific outreach involving people with disabilities does not require specific techniques for specific conditions. An \\ inclusive approach involving complementary senses not only helps those with disabilities but everyone else as well.
}

\author{
Enrique Pérez-Montero
}

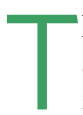
hose teaching and communicating scientific results should aim to reach all kinds of audiences to the utmost extent. Nevertheless, universality of the dissemination of knowledge encompassing all scientific fields is still far away. Indeed, some groups with varying degrees of disability have been traditionally marginalized from the complete learning and outreach of these matters. Instead, when these groups reach a high education level they are usually oriented towards humanity careers or other very specific applications - for instance the case of physiotherapy for some visually impaired people. As a result, the ratio of students with any level of disability studying for careers related to scientific research is much lower than their number in society would suggest. For this reason, it is key to raise the awareness of teachers and scientists about the need to use strategies and methodologies that make their efforts more inclusive.

One of the most important obstacles that we find when designing an educational project at any level, or a strategy for outreach in a research centre, is the diversity of the different disabilities - including motor, sensorial or intellectual - of people who may need different solutions to interact with the environment and gain information about a given project. As an example, there is a great variety of visual diseases, affecting different aspects of vision, which makes it appropriate to treat the collective of the visually disabled as not being totally blind. Their remaining visual capacity is functional in many cases although they can be blind in the centre of the field of vision, its periphery or show sensitivity to light and colours, and so on. In addition, each person has different previous abilities, experiences, education and background.

Nevertheless, specificity of visual ability should not to be translated into differentiated educational or outreach programmes, which would necessarily imply greater costs and attention, which would in turn make these efforts more unavailable for many teaching and outreach teams on limited resources. Instead, it is much more appropriate to design inclusive solution strategies for everybody at the same time.

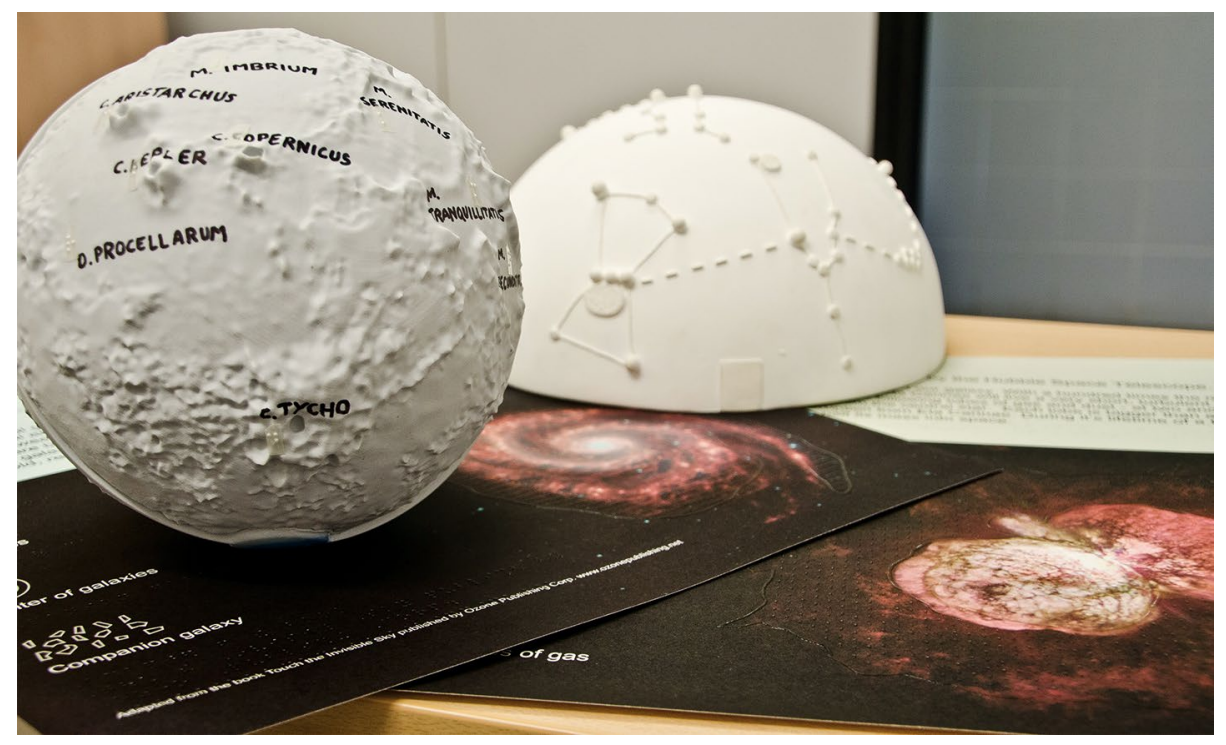

Fig. 1| 3D tactile models. The semi-sphere of the constellations (right) from the planetarium show El Cielo en tus Manos (The Sky in Your Hands) and a tactile globe of the Moon (left) are part of a growing collection of tactile models developed for the project $A$ Touch of the Universe that can be printed on 3D printers worldwide. Credit: photo, Amelia Ortiz-Gil (University of Valencia Astronomical Observatory); prints visible in photo, NASA/CXC/SAO

The application of these new methods for including all kinds of disabled people also produces very satisfactory results for nondisabled people, whose learning is reinforced as a consequence of the use of other sensorial channels for the acquisition of the information, engaging them in the learning process when disabled people are also present. This inclusive and non-excluding teaching approach is being applied at several levels of basic education and, to some extent, in certain museums with moderate success. Nevertheless, its application is still very low in the context of science curricula for highlevel education and outreach carried out by institutions and media.

In the concrete case of astronomy, it can be wrongly assumed that teaching and outreach cannot be universal because of the need to show results using images, animations, graphic representations and so on, which forcibly excludes all groups with some kind of visual disability from the total comprehension of different astronomical concepts. For this reason, many researchers and communicators around the world are making a special effort to design and construct content and materials especially adapted for visually disabled people. These innovations help them to understand concepts through the use of their hands, such as books in Braille or 3D relief models of different celestial objects or the night sky. For example, Fig. 1 shows models from A Touch of the Universe - a tactile astronomy kit for educators of children who are visually impaired. It includes 3D models of the rocky planets, with a soundtrack from a planetarium show and books in Braille. There is also an online webinar to train public communicators and teachers on how to use those resources.

I'm a blind researcher at the Instituto de Astrofísica de Andalucía in Granada, Spain, and in 2014 I started the Astroaccesible project. The main objective of this outreach project is to improve the teaching and popularization of astronomy among blind and visually disabled people by helping teachers and scientists to be more conscious 
Box 1 | Some simple dos and don'ts for improving access and clarity for audiences with or without sensorial disabilities

1. Don't abuse white backgrounds. Most people tend to use white backgrounds simply because we are used to reading on white sheets, but nowadays most computers and projectors emit light at a luminosity and frequency that tire the eyes very quickly. In addition, people affected with photophobia or extreme sensitivity to light won't be able to access the information.

2. Use high-contrast fonts. Using coloured fonts helps to improve the visibility of the text. Bold fonts hugely help to make text more readable.

3. Clearly identify the titles. People who cannot clearly hear the voice of the speaker will appreciate that the subject is clearly identified on all slides in order to know if a change has occurred during the presentation.

4. Describe all images and graphics. People who cannot visually access the slides will need an oral description of those images relevant for the presentation. In the case of graphics, it will be necessary to say what the axes represent and what we want to express with the plot.

5. Always look towards the audience. Some people need to read the lips of the speaker to follow the presentation. It is preferable to have the screen of the computer in front of the speaker. Try not to speak when you are looking at the back screen. In addition, if a microphone is not being used, speaking while facing the audience also will help to improve the clarity of the sound.

6. Provide subtitles and audio descriptions. When using video or audio, it is convenient to add subtitles for deaf and voice descriptions for blind audiences. Don't forget the credits if they are relevant.

7. Use big sizes for the images. Don't use just one slide to show many images. Add new slides if necessary to ensure the images are visible. Don't saturate the space with long text either.

8. Give alternatives to the pointer. The use of a pointer is a good resource that helps the speaker to indicate some aspects of the slides. However, if possible, it must be reinforced by an oral explanation of what it is being indicated, avoiding expressions such as "here" or "there".

of the need to extend their activities to all groups by being more inclusive and by providing advice and expertise on the heterogeneity of the diseases of the eye.

Among the different strategies to make all types of explanations more inclusive, the most straightforward is to give more complete descriptions of all of the images and animations presented. Some simple but effective tips are outlined in Box 1. The inclusivity of all activities is guaranteed in this way as the visual information is still used, just adapted in order to complement the activities, and its use is always complementary and not compulsory. Sometimes using high-contrast or different image sizes can also help those able to see specific visual patterns. Given that nowadays new technologies supply solutions to transform any written text into voice, it is not crucial to transform the information that we want to share into any specific code such as Braille. In addition, sheets that can be touched, 3D models and sounds to reinforce some of the information can also be used as resources not only to reach out to everybody but also to make all of the content more attractive to everybody, as even when using any kind of visual information some abstraction exercise must be done.

Many of the activities carried out over the past few years in the framework of Astroaccesible have a face-to-face character and range from workshops and conferences in centres specialized in the treatment of people with disabilities or in educational centres with or without the presence of impaired students. They have also provided assistance at meetings and congresses with the aim of increasing the sensitivity of scientists and outreach technicians to adopt more inclusive strategies and methods. Of special interest are activities devoted to showing and describing in situ (with the required adaptations) such places related to the science being discussed. For example, we arranged a visit to the Calar Alto Observatory (Almería, Spain), which included a visit to the control room and the dome of its 3.5-m telescope. This visit was prepared using tactile sheets showing the map of the observatory and the elevation of the telescope buildings. Another similar excursion was made to the Tabernas Desert (Almería, Spain) with a group of around 30 people with different degrees of visual impairment. This place has some similarities with the surface of the planet Mars, as it presents at different scales the traces of turbulent water on a landscape that is now dry and arid. This activity was produced with the help of a group of geologists. The design and printing of 3D reproductions of the ground and of the planet Mars helped to transmit in a very efficient way basic concepts about the history of the Solar System.

On a larger scale, however, teaching and outreach experiences carried out at different educational levels and research institutes remain modest in the context of science, and the commitment from all institutions must be strengthened if a nearfuture objective of inclusion is to be reached for all disabled people in the different fields of scientific knowledge. Adopting inclusive methodologies does not only help to reach this goal but would also boost the actual teaching and outreach efforts for all members of the public.

Enrique Pérez-Montero

Instituto de Astrofísica de Andalucía, Consejo Superior de Investigaciones Cientificas, Granada, Spain. e-mail:epm@iaa.es

Published online: 7 February 2019 https://doi.org/10.1038/s41550-019-0693-3

\section{Acknowledgements}

E.P.-M. has written this Comment on behalf of the International Astronomical Union Working Group for Equity and Inclusion. He acknowledges A. Ortiz-Gil and the Astronomical Observatory of the Universidad de Valencia for kindly supplying much of the material used in the activities of the Astroaccesible project. He also thanks the Outreach Office of the Instituto de Astrofísica de Andalucía and the partners of the Association Ciencia sin Barreras. 